\title{
Effects of Depression, Anxiety, Stigma, and Disclosure on Health-Related Quality of Life among Chronic Hepatitis B Patients in Dalian, China
}

\author{
Ge Li, ${ }^{1 *}+$ Gongchen Wang, ${ }^{2} \dagger$ Fang-Chi Hsu, ${ }^{3}$ Jianzhao Xu, ${ }^{4}$ Xia Pei, ${ }^{2}$ Bo Zhao, ${ }^{2}$ and Avinash Shetty ${ }^{5}$ \\ ${ }^{1}$ Department of Precision Medicine, Wake Forest University School of Medicine, Winston-Salem, North Carolina; ${ }^{2}$ Department of Infectious \\ Disease, Dalian Sixth People Hospital, Liaoning, China; ${ }^{3}$ Division of Public Health, Department of Biostatistics and Data Science, \\ Wake Forest School of Medicine, Winston-Salem, North Carolina; ${ }^{4}$ Department of Biochemistry, Wake Forest University School of Medicine, \\ Winston-Salem, North Carolina; ${ }^{5}$ Office of Global Health, Department of Pediatric/Infectious Disease, Wake Forest School of Medicine, \\ Winston-Salem, North Carolina
}

\begin{abstract}
Chronic hepatitis B virus (HBV) infection is a major public health problem in China. We evaluated the impact of psychosocial factors (stigma, disclosure, depression, and anxiety) on health-related quality of life (HRQoL) among people living with chronic HBV infection (CHB) in the city of Dalian, Liaoning Province, China. In this hospital-based crosssectional study, 401 patients living with chronic HBV infection were enrolled as study participants. Study measures included the Beck depression and anxiety inventory, the WHO Quality of Life (WHOQOL-BREF) assessment, the Toronto Chinese HBV Stigma Scale, and disclosure of HBV status to sexual partners. The primary outcome was HRQoL score as measured by the WHOQOL-BREF. A linear regression model was used to examine the association between HRQoL and the potential risk factors including stigma, disclosure, depression, anxiety, and sociodemographic variables. Stigma, disclosure, depression, and anxiety were the covariates of interest. A majority of the participants were females $(n=251$, $65.6 \%$ ), married (81.6\%), and had a college or higher degree (32.4\%). Depression, anxiety, stigma, and disclosure of HBV infection were associated with low HRQoL in all four domains of the WHOQOL-BREF (physical, psychological, social, and environmental domains) $(P<0.05)$, when all psychological factors were included in the model separately. Depression was found to be independently associated with low HRQoL in people living with HBV, when all psychological factors were included in the model simultaneously $(P<0.0001)$. Our data indicate the urgent need for healthcare providers $(\mathrm{HCPs})$ and policy-makers to implement psychological interventions to improve HRQoL among people living with $\mathrm{CHB}$.
\end{abstract}

\section{INTRODUCTION}

Chronic hepatitis B infection (CHB) caused by the hepatitis $B$ virus (HBV) is a major global public health problem resulting in considerable morbidity and mortality. An estimated 280 million people are chronically infected with HBV; in 2015, more than 850,000 deaths were reported primarily because of complications of HBV, including cirrhosis and liver cancer. ${ }^{1}$ China historically has borne the brunt of HBV infection burden in the world. Current estimates indicate that $6 \%$ of the population (i.e., 90 million people) in China are living with $\mathrm{CHB}^{2}$ Chronic HBV infection is associated with high mortality because of progressive complications from cirrhosis, hepatocellular carcinoma, and liver failure. The burden of $\mathrm{CHB}$ results in substantial economic burden on their families and the society. ${ }^{3}$ Like other chronic disease, patients with $\mathrm{CHB}$ face a number of challenges to maintain physical, psychological, and social health. ${ }^{4-8}$ The assessment of health-related quality of life (HRQoL) is an important component in the management of $\mathrm{CHB}$ patients. ${ }^{9,10}$

Despite national HBV antidiscrimination laws and regulations in China, patients living with CHB commonly experience discrimination in many settings including workplace, educational institutions, healthcare locations, and in the community. ${ }^{9,11,12}$ Stigma may be associated with feelings of shame, blame, fear, anxiety, depression, and lower quality of life (QOL) among people living with $\mathrm{CHB} .{ }^{11-13}$ To our knowledge, disclosure issues related to HBV infection and its impact on HRQoL have not been studied previously in China. Previous studies found that comorbid depression is common among patients with hepatitis-related cirrhosis or liver failure caused by hepatitis B or $\mathrm{C} .{ }^{14,15}$ Although

${ }^{*}$ Address correspondence to $\mathrm{Ge} \mathrm{Li}$, Department of Precision Medicine, Wake Forest University School of Medicine, WinstonSalem, NC 27157. E-mail: gli@wakehealth.edu

†These authors contributed equally to this work. studies have investigated the relationship between HRQoL and $\mathrm{CHB}$ infection including disease progression, data on the impact of multiple psychosocial factors on HRQoL are very limited.

The objective of this cross-sectional study was to assess the impact of psychosocial factors (stigma, disclosure, depression, and anxiety) on HRQoL among people living with $\mathrm{CHB}$ in the city of Dalian, China.

\section{METHODS}

Study setting. This cross-sectional study was conducted at the Dalian Sixth People's Hospital, affiliated with Dalian Medical University, which is one of the largest infectious disease hospitals in the three provinces in northeastern China. Founded in 1950, the hospital now has 600 beds and 17 clinical departments. The hospital incorporates and integrates clinical services for infectious disease in conjunction with medical education and research programs. Dalian is a major seaport city in the south of Liaoning Province, which has a population of 6.17 million. Patients seeking care at Dalian Medical University are primarily from the urban area of Dalian, although some patients are referred from rural areas. Most study participants were recruited through the department of liver disease at Dalian Medical University.

Eligibility criteria. The diagnostic standards and classification of $\mathrm{CHB}$ in our patients were informed by the Guidelines for Prevention and Treatment of Chronic Hepatitis B (China, 2015 version) published by the Chinese Society of Hepatology, Chinese Medical Association (CMA), and the Society of Infectious Diseases, CMA. ${ }^{16,17}$ The diagnosis of compensated cirrhosis was based on imaging findings of ultrasound or computed tomography (CT). Decompensated cirrhosis was defined as having serious complications of cirrhosis, including ascites, variceal bleeding, or hepatic encephalopathy. 
The inclusion criteria included participants aged $\geq 18$ years, residing in the study catchment area for $>6$ months, and living with a diagnosis of $\mathrm{CHB}$ or HBV-related cirrhosis. The diagnosis of $\mathrm{CHB}$ was determined by positive hepatitis $\mathrm{B}$ surface antigen and/or HBV DNA for $>6$ months, with increased levels of serum alanine aminotransferase.

Patients were excluded if they had cirrhosis coinfected with HIV, hepatitis $C$, or hepatitis $D$, received interferon therapy, had chronic lung disease, were suffering from alcoholic or drug-induced liver disease, tuberculosis, severe psychiatric disorders, or were pregnant.

Sample size. We enrolled a convenience sample of 430 patients living with $\mathrm{CHB}$ attending inpatient or outpatient clinics at Dalian Sixth People's Hospital. Given this sample size, we would have $80 \%$ power to detect a correlation of 0.14 between HRQoL scores and psychological risk factors. ${ }^{18}$ The study recruitment started in January 2017 and ended by December 2017. The participants were consecutively selected in the order of their clinic appointment. The sampling process was ended when the desired total amount of participants was reached.

Study procedures. Each participant completed a semistructured questionnaire in the native Chinese language (Mandarin). Data from 29 of 430 participants were excluded because less than $85 \%$ of the survey items were completed.

Before enrolling, CHB patients in the hepatology clinic underwent screening by a trained medical staff member to assess eligibility and interest in study participation. Those who were eligible and interested were provided detailed information about the study and given the opportunity to ask questions. Once informed written consent was obtained, participants were asked to complete the questionnaires. Validated questionnaires were administered in the native Chinese language. The English versions of the stigma and disclosure questionnaires were translated from English to Chinese by language experts and translated back to English by another set of language experts to ensure validity and consistency.

Ethical considerations. The Institutional Review Board of Wake Forest School of Medicine as well as Dalian Sixth People's Hospital affiliated with Dalian Medical University approved the study protocol. Written informed consent was obtained from all study participants. During the administration of the survey, patients with suspected severe psychiatric disorder including suicidal thoughts were immediately referred for psychiatric evaluation and treatment at the Dalian Mental Health Center (Dalian Seventh People's Hospital).

Study Instruments. Stigma scale. We modified the validated Toronto Chinese HBV Stigma Scale to measure stigma attitudes with $\mathrm{HBV}$ infection. ${ }^{19}$ The 12 -item stigma scale was derived and modified from HIV/AIDS stigma scales published in the literature. The internal consistency reliability of the 20 -item stigma scale (Cronbach's alpha) was $0.90 .{ }^{19}$ Participants were asked to provide their best response to a 1-point scale (each item on the questionnaire ranged from 0 to 1 [ $1=$ not at all a factor \{answer no and $0=$ very likely a factor \{answer yes\}]). The score range was $0-12$. Higher scores indicate greater levels of HBV stigma.

Disclosure scale. To determine disclosure or nondisclosure of CHB status among study participants, we modified the validated 15-item scale adapted from Derlega et al., ${ }^{20}$ which was originally used to examine the reasons why men living with HIV disclose or not disclose their HIV serostatus to their casual sexual partners. ${ }^{21}$ A clear factor structure was used for disclosure questionnaires. We modified the disclosure scale that was composed of 12 items and four factor solutions (three items per factor), including responsibility, instruction, relationship consequences, and emotional relations. Each item was scored $(0=$ no and $1=$ yes). Each factor score ranged from 0 to 3 . The total score ranged from 0 to 12 . The disclosure score reflected the patients' attitudes toward disclosure of their positive HBV infection status to family members, friends, colleagues, or community members. The internal consistency reliability (Cronbach's alpha) of the disclosure scale (20 items and four factor solutions) was as follows: responsibility ( $\alpha=$ $0.57)$, instruction $(\alpha=0.76)$, relationship consequences $(\alpha=$ $0.90)$, and emotional release $(\alpha=0.69) .{ }^{21}$

Beck depression inventory. The validated Beck Depression Inventory (BDI) is a frequently used "self-reported" scale developed by Beck et al. ${ }^{22}$ to measure emotional, cognitive, somatic, and motivational components. The Beck scale is composed of 21 items. Every question scored from " 0 " to " 3 ," for a total score ranging between " 0 " and "63." Results between 0 and 9 are defined as minimal depression, $10-18$ as mild depression, 19-29 as moderate depression, and 30-63 as severe depression. ${ }^{22}$ The internal consistency reliability of the validated BDI was excellent (Cronbach's alpha of 0.91).

Beck anxiety scale. The validated Beck Anxiety Scale (BAS), developed by Beck and others, ${ }^{23}$ is a self-evaluation scale designed to establish the frequency of anxiety symptoms in individuals. The scale is composed of 21 items scored between " 0 " and " 3 ," with rankings of "absent," "mild," "moderate," and "severe." Scores range between " 0 " and "63." Results between 0 and 9 are categorized as minimal anxiety, 10-16 as mild anxiety, 17-29 as moderate anxiety, and 30-63 as severe anxiety. ${ }^{23}$ The BAS was adapted to evaluate anxiety among Chinese doctors. ${ }^{24}$ The internal consistency reliability of the validated BAS used in our study was excellent (Cronbach's alpha of 0.93).

The WHO quality of life measure. The WHO Quality of Life (WHOQOL) project was initiated in 1991. The aim was to develop an international cross-culturally comparable QOL assessment instrument to assess an individual's perceptions in the context of his or her culture and value systems, and his or her personal goals, standards, and concerns. ${ }^{25,26}$ The WHOQOL instruments were developed collaboratively in a number of centers worldwide and have been widely field-tested. The WHOQOL-BREF instrument comprises 26 items, which measure the following broad domains: physical health, psychological health, social relationships, and environment. ${ }^{26}$ The WHOQOL-BREF is a shorter version of the original instrument that is more convenient for use in large research studies or clinical trials. The scores scale in a positive direction, with a higher score indicating higher QOL as measured by the WHOQOL-BREF. ${ }^{27}$ Our study showed acceptable internal consistency of the scale for the domains of physical health $(\alpha=$ $0.87)$, psychological health $(\alpha=0.91)$, social relationships $(\alpha=$ $0.88)$, and environment ( $\alpha=0.93$ ).

Statistical analysis. Statistical analyses were completed using SAS software version 9.4 (SAS Institute, Cary, NC). Summary statistics including means and SD were computed for the continuous characteristics, and counts and percentages were computed for discrete characteristics. The primary outcome was HRQoL score as measured by the WHOQOLBREF. The distribution of this score was checked and 
transformed to satisfy normality assumption as necessary. A linear regression model was used to examine the association between HRQoL and the potential risk factors including stigma, disclosure, depression, anxiety, and other social demographic factors. Stigma, disclosure, depression, and anxiety were the covariates of interest. Age, gender, education, medical insurance, cirrhosis, alcohol drinking, other chronic diseases, stage, and years of diagnosis were known risk factors and were adjusted in the model. The covariates of interest were fitted one at a time, and fitted jointly in the model. The interactions between psychological factors and education (college and above, high school, and middle school and below), other chronic diseases (yes and no), stage (cirrhosis + carcinoma and chronic hepatitis $B)$, and years of diagnosis ( $\geq 9$ and $<9$ ) were tested additionally by including the interaction term in the model one at a time. To ensure we had enough sample size in each group, the cirrhosis and carcinoma groups were combined. The years of diagnosis were dichotomized based on the median (9 years). Stratified analysis was also performed. Note that if the interaction term was significant, meaning the effect was different between each stratum, we then interpreted the stratified result. If the interaction term was not significant, even though the stratified result might be significant, we did not interpret the stratified result. Model diagnosis including residual plots, outlier detection, and collinearity statistics was performed.

\section{RESULTS}

Demographic characteristics. The sociodemographic information of the participants is shown in Table 1. In total, 401 individuals completed and returned the survey (response rate, $93 \%)$. Participants were predominantly female $(n=251$, $65.6 \%)$. The majority of the patients $(81.6 \%)$ were married, with a mean age of 47 years (SD, 13). There were 130 (32.4\%) participants who had a college degree or above.

Among the 401 participants, 375 were diagnosed with chronic hepatitis B infection (CHB, 93.8\%), and 246 (66.1\%) were diagnosed with $\mathrm{CHB}$ including cirrhosis. The mean years since diagnosis of CHB were 10.7 (SD is 9.5) years. Among the 401 participants, 295 did not have other chronic diseases, and 106 (26.4\%) had other chronic diseases.

Depression, anxiety, disclosure, stigma, and QOL scores. Summary statistics for psychological variables including depression, anxiety, disclosure, nondisclosure, and stigma variables as well as four WHOQOL-BREF domains of HRQoL measures are shown in Table 2. Among the 401 participants, the BDI mean score was 9.12; the maximum score was 46 . The BAS mean score was 8.26; the maximum score was 53 . The BDI and BAS scores reflect the severity of the symptoms. The mean score of stigma is shown in Table 2, with higher scores reflecting a greater level of severity. The mean score of disclosure 1 (responsibility) was 1.98, disclosure 2 (instruction) was 2.22, disclosure 3 (relationship consequences) was 1.99, and disclosure 4 (emotional release) was 1.69. The mean score of the WHOQOL-BREF was 22.78 for the physical domain, 19.57 for the psychological domain, 10.31 for the social domain, and 26.25 for the environmental domain.

Associations of depression, anxiety, disclosure, and stigma with QOL. The results from the multivariable linear regression models, association between psychological factors
TABLE 1

Demographic characteristics of participants

\begin{tabular}{|c|c|c|}
\hline & $(n=401)$ & Mean/SD (\%) \\
\hline Age (years) & & $47.0 \pm 13.0$ \\
\hline Gender (female) & 251 & 65.6 \\
\hline \multicolumn{3}{|l|}{ Education } \\
\hline College and above & 130 & 32.4 \\
\hline High school & 126 & 31.4 \\
\hline Middle school & 122 & 30.4 \\
\hline Primary school & 20 & 5.0 \\
\hline Illiteracy & 3 & 0.8 \\
\hline \multicolumn{3}{|l|}{ Medical insurance } \\
\hline Social health insurance & 304 & 75.8 \\
\hline Public expense & 18 & 4.5 \\
\hline New rural cooperative & 46 & 11.5 \\
\hline Own expense & 20 & 5.0 \\
\hline Commercial medical insurance & 7 & 1.8 \\
\hline Others & 6 & 1.5 \\
\hline \multicolumn{3}{|l|}{ Marriage status } \\
\hline Married & 327 & 81.6 \\
\hline Single & 48 & 12.0 \\
\hline Divorced or widowed & 26 & 6.5 \\
\hline $\begin{array}{l}\text { Years diagnosed with HBV } \\
\text { Hepatitis B stage }\end{array}$ & & $10.7 \pm 9.5$ \\
\hline Chronic hepatitis B & 375 & 93.8 \\
\hline Chronic hepatitis B with cirrhosis & 246 & 61.1 \\
\hline Hepatic carcinoma & 2 & 0.5 \\
\hline Drink (yes) & 50 & 12.5 \\
\hline \multicolumn{3}{|l|}{ Other chronic diseases } \\
\hline Have chronic diseases ${ }^{\star}$ & 106 & 26.4 \\
\hline No chronic diseases & 295 & 73.6 \\
\hline $\begin{array}{l}\text { See doctor in the last } 12 \text { months because } \\
\text { of HBV }\end{array}$ & 188 & 46.9 \\
\hline $\begin{array}{l}\text { See doctor if follow-up service is provided } \\
\text { (yes) }\end{array}$ & 273 & 68.6 \\
\hline
\end{tabular}

chronic diseases.

(depression and anxiety), disclosure, nondisclosure, stigma, and QOL domain (physical, psychological, social, and environment), are listed in Table 3. After adjusting for sociodemographic and other variables (age, gender, education [college and above versus high school, high school versus middle school, and middle school and below], medical insurance, cirrhosis, alcohol consumption, other chronic diseases, $\mathrm{CHB}$ stage, and years with diagnosis of $\mathrm{CHB}$ ), we found that the participants' HRQoL in the physical domain had a significantly negative association with depression $(P<$ $0.0001)$, anxiety $(P<0.0001)$, and stigma $(P=0.0142)$. Participants' HRQoL in the psychological domain had a significantly negative relationship with depression $(P<0.0001)$, anxiety $(P<0.0001)$, and disclosure factors: relationship consequences $(P=0.0086)$, emotional release $(P=0.0069)$, and stigma $(P=0.0094)$. Participants' HRQoL in the social domain had a significantly negative association with depression $(P<0.0001)$, anxiety $(P<0.0001)$, and disclosure factor: emotional release $(P=0.0132)$ and stigma $(P=$ 0.0041). Participants' HRQoL in the environmental domain had a significantly negative relationship with depression $(P<$ $0.0001)$, anxiety $(P<0.0001)$, and disclosure factors: relationship consequences $(P=0.0025)$, emotional release $(P=$ $0.0122)$, and stigma $(P=0.0154)$. The results from Table 3 reveal that all four factors of psychosocial morbidity (depression, anxiety, stigma, and disclosure HBV infection) had a negative relationship with the HRQoL physical, psychological, social, and environmental domains measured by the 
TABLE 2

Summary statistics for psychological variables and quality of life measures

\begin{tabular}{|c|c|c|c|c|c|c|}
\hline Variable & $N$ & Mean & $\mathrm{SD}$ & Median & Minimum & Maximum \\
\hline score_depression & 401 & 9.12 & 9.28 & 6 & 0 & 46 \\
\hline score_anxiety & 401 & 8.26 & 9.13 & 5 & 0 & 53 \\
\hline score disclosure1_responsibility & 400 & 1.95 & 1 & 2 & 0 & 3 \\
\hline score disclosure2_instruction & 401 & 2.19 & 1.08 & 3 & 0 & 3 \\
\hline $\begin{array}{l}\text { score disclosure3_relationship } \\
\text { consequences }\end{array}$ & 401 & 1.9 & 1.21 & 2 & 0 & 3 \\
\hline score disclosure4_emotional release & 401 & 1.66 & 1.13 & 1 & 0 & 3 \\
\hline Nondisclosure_total & 401 & 7.88 & 3.36 & 8 & 0 & 12 \\
\hline score_stigma & 399 & 3.77 & 2.38 & 3 & 0 & 12 \\
\hline score_physical & 398 & 22.78 & 4.37 & 22.5 & 10 & 34 \\
\hline score_psychological & 401 & 19.57 & 4.21 & 20 & 6 & 30 \\
\hline score_social & 400 & 10.31 & 2.75 & 10 & 0 & 15 \\
\hline score_enviromental & 400 & 26.25 & 7.12 & 26 & 0 & 40 \\
\hline
\end{tabular}

WHOQOL-BREF. The results from unadjusted models are presented in Supplemental Table 1.

After adjusting for age, gender, medical insurance, cirrhosis, combination with other chronic diseases, and years with diagnosis of $\mathrm{CHB}$, higher education level had a significantly positive association with HRQoL for all four domains (Table 4). Those with higher education levels were more likely to have higher HRQoL.

Previously, each psychological factor was fitted in the model one at a time. A multivariable model of all psychological factors (depression, anxiety, stigma, and disclosure of HBV infection) was used to evaluate independent effects of each psychological factor independence on HRQol. The association result, after adjusting for age, gender, education (college and above versus high school and high school versus middle school), medical insurance, cirrhosis, other chronic diseases, and year of diagnosis, is presented in Table 5. The participants' HRQoL in all domains continued to have a significantly negative association with depression (physical $P<0.0001$, psychological $P<0.0001$, social $P<0.0001$, and environment $P<0.0001)$. Depression, as an independent factor, was significantly associated with HRQoL in CHB patients. No relationship was observed between stigma and disclosure of HBV infection related with HRQoL domain under this model. The unadjusted result is presented in Supplemental Table 2.

The significant interaction $P$-values show that the associations between stigma and physical and psychological HRQoL were different between the two groups for years of diagnosis ( $\leq 9$ versus $>9$ ) (interaction $P=0.0366$ and 0.0438 , respectively). The negative association between stigma and HRQoL (physical and psychological) was only seen in those participants with years of diagnosis $\leq 9$. There was no association between stigma and physical and psychological HRQoL in those participants with years of diagnosis $>9$ (Supplemental Table 3). In addition, the negative association between disclosure (1 and 4) and HRQoL (social and environment) was only seen in those participants with years of diagnosis $\leq 9$. The negative association between disclosure (4) and social HRQoL was only noted in those participants with comorbidities (Supplemental Table 4). The negative association between depression and environmental HRQoL was only seen for those participants with CHB (Supplemental Table 5). The negative association between anxiety and social and environmental HRQoL was only observed in those participants with college and above education (Supplemental Table 6).

\section{DISCUSSION}

In this study, we evaluated the impact of psychosocial factors (stigma, disclosure, depression, and anxiety) on HRQoL among people living with $\mathrm{CHB}$ in China. Many studies have reported that people living with chronic hepatitis have psychiatric disorders that can affect their HRQoL. ${ }^{28,29} \mathrm{De}-$ pression and anxiety are the most frequent mental disorders in $\mathrm{CHB}$ patients in China. ${ }^{30}$ One study from Turkey reported a

TABLE 3

Multivariable association between psychological factors and quality of life-psychological factors are included in the model one at a time

\begin{tabular}{|c|c|c|c|c|c|c|c|c|c|c|c|c|}
\hline & \multicolumn{3}{|c|}{ Physical } & \multicolumn{3}{|c|}{ Psychological } & \multicolumn{3}{|c|}{ Social } & \multicolumn{3}{|c|}{ Environmental } \\
\hline & Estimate & $\begin{array}{l}\text { Standard } \\
\text { error }\end{array}$ & $P$-value & Estimate & $\begin{array}{l}\text { Standard } \\
\text { error }\end{array}$ & $P$-value & Estimate & $\begin{array}{l}\text { Standard } \\
\text { error }\end{array}$ & $P$-value & Estimate & $\begin{array}{l}\text { Standard } \\
\text { error }\end{array}$ & $P$-value \\
\hline score depression & -0.20 & 0.02 & $<0.0001$ & -0.2 & 0.02 & $<0.0001$ & -0.11 & 0.01 & $<0.0001$ & -0.31 & 0.03 & $<0.0001$ \\
\hline score anxiety & -0.11 & 0.02 & $<0.0001$ & -0.11 & 0.02 & $<0.0001$ & -0.07 & 0.01 & $<0.0$ & -0.25 & 0.03 & $<0.0001$ \\
\hline $\begin{array}{l}\text { score disclosure1__ } \\
\text { responsibility }\end{array}$ & -0.11 & 0.20 & 0.5663 & -0.21 & 0.19 & 0.2513 & -0.17 & 0.13 & 0.1636 & -0.40 & 0.32 & 0.2166 \\
\hline score disclosure2_instruction & -0.11 & 0.18 & 0.5720 & -0.15 & 0.18 & 0.4183 & -0.13 & 0.11 & 0.2576 & -0.45 & 0.30 & 0.1339 \\
\hline $\begin{array}{l}\text { score disclosure3- } \\
\text { relationship consequences }\end{array}$ & -0.30 & 0.12 & 0.0114 & -0.30 & 0.11 & 0.0086 & 3 & 0.07 & 0.0755 & -0.57 & 0.19 & 0.0025 \\
\hline $\begin{array}{l}\text { score disclosure4_emotional } \\
\text { release }\end{array}$ & -0.8 & 0.18 & 0.1227 & -0.47 & 0.17 & 0.0069 & -0.28 & 0.11 & 0.0132 & -0.73 & 0.29 & 0.0122 \\
\hline score nondisclosure & -0.07 & 0.06 & 0.2590 & 0.0 & 0.02 & 0.6441 & -0.0 & 0.0 & 0.2263 & -0.12 & 0.1 & 0.2238 \\
\hline score stigma & -0.21 & 0.08 & 0.0142 & -0.22 & 0.08 & 0.0094 & -0.16 & 0.05 & 0.0041 & -0.34 & 0.14 & 0.0154 \\
\hline
\end{tabular}

Adjusting for age, gender, education (college and above vs. high school vs. middle school and below), medical insurance, liver cirrhosis, alcohol drinking, other chronic diseases, stage, and years of diagnoses. 
TABLE 4

Multivariable association between demographic factors and quality of life

\begin{tabular}{|c|c|c|c|c|c|c|c|c|c|c|c|c|}
\hline \multirow[b]{2}{*}{ Parameter } & \multicolumn{3}{|c|}{ Physical } & \multicolumn{3}{|c|}{ Psychological } & \multicolumn{3}{|c|}{ Social } & \multicolumn{3}{|c|}{ Environmental } \\
\hline & Estimate & $\begin{array}{l}\text { Standard } \\
\text { error }\end{array}$ & $P$-value & Estimate & $\begin{array}{l}\text { Standard } \\
\text { error }\end{array}$ & $P$-value & Estimate & $\begin{array}{l}\text { Standard } \\
\text { error }\end{array}$ & $P$-value & Estimate & $\begin{array}{l}\text { Standard } \\
\text { error }\end{array}$ & $P$-value \\
\hline Age & -0.03 & 0.02 & 0.1808 & -0.04 & 0.02 & 0.0416 & -0.01 & 0.01 & 0.6469 & -0.06 & 0.03 & 0.0862 \\
\hline Gender (female vs. male) & -0.15 & 0.46 & 0.75 & -0.74 & 0.44 & 0.0952 & -0.47 & 0.29 & 0.1098 & -1.03 & 0.74 & 0.1660 \\
\hline $\begin{array}{l}\text { Education (college and above vs. } \\
\text { middle school and below) }\end{array}$ & 2.37 & 0.60 & $<0.0001$ & 1.95 & 0.57 & 0.0007 & 1.07 & 0.38 & 0.0047 & 3.85 & 0.95 & $<0.0001$ \\
\hline $\begin{array}{l}\text { Education (high school vs. middle } \\
\text { school and below) }\end{array}$ & 0.88 & 0.53 & 0.0996 & 0.80 & 0.51 & 0.1150 & 0.26 & 0.34 & 0.4326 & 1.45 & 0.85 & 0.0897 \\
\hline Medical insurance (yes vs. no) & 0.35 & 0.53 & 0.5137 & 0.06 & 0.51 & 0.9048 & 0.31 & 0.34 & 0.3646 & 1.07 & 0.85 & 0.2107 \\
\hline Liver cirrhosis (yes vs. no) & -0.89 & 0.49 & 0.0733 & -1.04 & 0.47 & 0.0271 & -0.69 & 0.31 & 0.0282 & -1.37 & 0.79 & 0.0839 \\
\hline Alcohol drinking (yes vs. no) & -0.11 & 0.69 & 0.87 & -0.40 & 0.66 & 0.5402 & -0.17 & 0.44 & 0.6913 & -1.08 & 1.10 & 0.3277 \\
\hline Other chronic disease & -0.62 & 0.54 & 0.25 & -0.35 & 0.51 & 0.4969 & -0.72 & 0.34 & 0.0359 & -0.70 & 0.87 & 0.4227 \\
\hline $\begin{array}{l}\text { Stage (carcinoma and HBV cirrhosis } \\
\text { vs. chronic hepatitis B) }\end{array}$ & 0.42 & 0.90 & 0.6363 & -0.36 & 0.86 & 0.6797 & 0.22 & 0.57 & 0.6941 & -1.16 & 1.44 & 0.4218 \\
\hline Years diagnosed with HBV & -0.01 & 0.02 & 0.7150 & 0.01 & 0.02 & 0.6550 & 0.01 & 0.01 & 0.4837 & 0.01 & 0.04 & 0.6904 \\
\hline
\end{tabular}

high prevalence of depression with associated negative correlation between the physical environment and cultural areas on the BAS and WHOQOL-BREF. ${ }^{14}$ Our study found that depression and anxiety among people living with $\mathrm{CHB}$ were significantly associated with negative HRQoL in all four domains of the WHOQOL-BREF. In addition, the depression score was found to be associated with $H R Q O L$ in people living with $\mathrm{CHB}$, especially for social and environmental domains of HRQol.

In our study, when a multivariable association between demographic factors and quality of life was taken into consideration, we found the education level (college and above versus middle school and below) had a significantly positive association with HRQoL for all four domains of the WHOQOLBREF. This finding may be related to CHB patients' knowledge about HBV. Participants with a higher educational level had better knowledge about HBV transmission than people with a lower education level (e.g., below high school education), suggesting the importance of educating patients living with $\mathrm{CHB}$ about HBV and transmission risks. ${ }^{31}$ By contrast, our study showed a negative association between anxiety and social environmental QOL and participants with a higher education level (college and above) (Supplemental Table 6).
Although these participants were aware of HBV infection and transmission risks, they experienced challenges in coping with the diagnosis following confrontation with the disease, which may have more negative considerations related to cognition and psychological adjustment.

In this study, we also found that HBV-related stigma had negative consequences and adversely affected HRQoL in physical, psychological, social, and environmental domains. The results of the study showed a negative association between stigma and HRQoL (physical and psychological) in those participants with years of diagnosis $\leq 9$. In the early stage of the disease, patients may have a greater fear of social stigma and may be more concerned about the acceptance of the diagnosis by their family, friends, and society. To our knowledge, this is the first report showing the relationship between stigma and HRQoL in China. One previous study from China surveying HBV-related stigma among chronically infected patients and uninfected patients reported a significant association of social and internalized stigma with HBV infection. ${ }^{32}$ Another study investigating the impact of HBV knowledge and stigma on screening in Canadian Chinese individuals found that HBV-related stigma was associated with reduced rates of HBV screening. ${ }^{19}$

TABLE 5

Multivariable association between psychological factors and quality of life-psychological factors are included in the model simultaneously

\begin{tabular}{|c|c|c|c|c|c|c|c|c|c|c|c|c|}
\hline \multirow[b]{2}{*}{ Parameter } & \multicolumn{3}{|c|}{ Physical } & \multicolumn{3}{|c|}{ Psychological } & \multicolumn{3}{|c|}{ Social } & \multicolumn{3}{|c|}{ Environmental } \\
\hline & Estimate & $\begin{array}{l}\text { Standard } \\
\text { error }\end{array}$ & $P$-value & Estimate & $\begin{array}{l}\text { Standard } \\
\text { error }\end{array}$ & $P$-value & Estimate & $\begin{array}{l}\text { Standard } \\
\text { error }\end{array}$ & $P$-value & Estimate & $\begin{array}{l}\text { Standard } \\
\text { error }\end{array}$ & $P$-value \\
\hline $\begin{array}{l}\text { score } \\
\text { depression }\end{array}$ & -0.16 & 0.02 & $<0.0001$ & -0.18 & 0.02 & $<0.0001$ & -0.10 & 0.01 & $<0.0001$ & -0.23 & 0.04 & $<0.0001$ \\
\hline score_anxiety & -0.04 & 0.02 & 0.1262 & -0.01 & 0.02 & 0.5590 & -0.02 & 0.016 & 0.2221 & -0.12 & 0.04 & 0.0025 \\
\hline $\begin{array}{l}\text { score_ } \\
\text { disclosure_1 }\end{array}$ & 0.08 & 0.24 & 0.7275 & 0.01 & 0.22 & 0.9695 & -0.06 & 0.15 & 0.6787 & 0.16 & 0.38 & 0.6565 \\
\hline $\begin{array}{l}\text { score_ } \\
\text { disclosure_2 }\end{array}$ & 0.11 & 0.22 & 0.6138 & 0.19 & 0.20 & 0.3559 & 0.05 & 0.14 & 0.6898 & -0.01 & 0.351 & 0.9713 \\
\hline $\begin{array}{l}\text { score_ } \\
\text { disclosure_3 }\end{array}$ & -0.14 & 0.12 & 0.2534 & -0.10 & 0.11 & 0.3831 & 0.01 & 0.07 & 0.8224 & -0.23 & 0.19 & 0.2275 \\
\hline $\begin{array}{l}\text { score_ } \\
\quad \text { disclosure_4 }\end{array}$ & -0.08 & 0.20 & 0.6753 & -0.30 & 0.18 & 0.1082 & -0.15 & 0.12 & 0.2106 & -0.36 & 0.31 & 0.2511 \\
\hline $\begin{array}{l}\text { score }_{-} \\
\text {nondisclosure }\end{array}$ & -0.01 & 0.06 & 0.7802 & 0.03 & 0.06 & 0.5318 & -0.01 & 0.04 & 0.6663 & -0.03 & 0.10 & 0.7710 \\
\hline score_stigma & -0.13 & 0.08 & 0.1194 & -0.14 & 0.08 & 0.0847 & -0.10 & 0.05 & 0.0627 & -0.17 & 0.13 & 0.1903 \\
\hline
\end{tabular}


Hepatitis B virus (HBV)-related stigma is frequently experienced among Asian immigrants in high-income countries. ${ }^{33} \mathrm{~A}$ common misconception among participants was that HBV could be transmitted when people share food, a view shared among many Chinese immigrants residing in North America. ${ }^{34,35}$ In our study, $66 \%$ of participants agreed that people living with HBV should not be allowed to work in certain areas, such as restaurants. About $75 \%$ of the participants stated that people living with HBV should be isolated from others to protect the public. About $35 \%$ of participants reported feeling uncomfortable sharing clothing worn previously by a person living with HBV. Thus, patient education about HBV is a key priority to address stigma and discrimination and misconceptions regarding HBV transmission.

Many reports have found that people living with HBV have a poor understanding of the mode of transmission of HBV infection, and this may lead people with HBV to not disclose their positive serostatus to their partners and/or not to seek care and remain in care after their diagnosis. ${ }^{31,36} \mathrm{~A}$ knowledge, attitude, and practice study about HBV and liver cancer conducted among healthcare providers (HCPs) and public health professionals in China found that HBV-related knowledge was inadequate even among highly trained providers. ${ }^{37}$ Lower HBV knowledge was associated with participants who reported poor preventive practices compared with those who reported sound preventive practices. ${ }^{37}$ Of the surveyed HCPs, $38 \%$ reported positive HBsAg test results to the patient's employer, and $25 \%$ reported these results to the patients' schools, indicating that HCPs may be unintentionally contributing to stigma and discrimination of people living with $\mathrm{CHB}$. Thus, concerns of HBVrelated stigma and discrimination may result in low uptake of hepatitis $B$ education, screening, immunization, and sound preventive practices among at-risk individuals, underscoring the importance of effective HBV education among $\mathrm{HCPs}^{37}$

In our study, about $83 \%$ of participants agreed they would feel pity for someone with HBV infection. Although the Chinese government took legislative action in 2007 to eliminate discrimination against people living with HBV when seeking employment and pursuing educational opportunities, ${ }^{38}$ people living with $\mathrm{CHB}$ in China continue to experience discrimination. ${ }^{31}$ Studies have found that individuals with $\mathrm{CHB}$ who had a higher education level (bachelor degree or more) experienced less discrimination compared with those with a lower educational level. ${ }^{31}$ In our study, about $68 \%$ of participants had an education below the college level. Individuals with $\mathrm{CHB}$ often experience significant financial hardships because of limited opportunities for education and career advancement, especially for people residing in rural settings with fewer resources for education, prevention, diagnosis, and treatment of $\mathrm{CHB}$. Our study demonstrates that when stigma and discrimination affect people living with $\mathrm{HBV}$, it is predominantly associated with negative HRQoL. Experiences of discrimination can result in negative emotions. ${ }^{31}$ Our data should inform physicians, nurses, and other health providers of the necessity to address the psychological burden of people living with $\mathrm{CHB}$ and offer them early screening and treatment. Provision of accurate and accessible information about HBV to communities is crucial, to significantly reduce stigma and discrimination and raise awareness of legal protection and support systems across the country. ${ }^{31}$

Disclosure of positive HBV status to sexual partners and family members remains a major challenge for people living with $\mathrm{HBV}^{32}$ Our study reveals that HRQoL-related psychological and social environment is significantly associated with disclosure of HBV infection. This finding was noted when time since diagnosis of HBV infection was $<9$ years. Among the disclosure factors, we found that relationship consequences and emotional release were significantly associated with HRQoL. Our results suggest that many patients living with CHB may be concerned or worry about other people's reaction.

Low HBV awareness and knowledge were reported as important factors for nondisclosure of HBV status to contacts in most reports, ${ }^{18,32,35}$ but not in others. ${ }^{31}$ Accordingly, educational interventions could be crucial to reducing HBV discrimination, raise awareness in the community regarding transmission of HBV, and dispelling myths and misconceptions, especially in rural communities. ${ }^{39}$ Community education and mobilization campaigns related to hepatitis $B$ vaccination are critical to preventing $\mathrm{CHB}$ infection and reducing stigma and discrimination toward people living with HBV. ${ }^{31}$

\section{LIMITATIONS}

This study has several limitations. First, some of the study participants also suffered from other chronic diseases that could have resulted in negative mood and increased negative psychological emotion. Second, the participants who consented to the study may have exhibited different health behaviors or resources compared with those who did not consent. Third, the survey questionnaires required 40-50 minutes to complete, thereby limiting the accuracy of responses. Fourth, our cross-sectional survey used a convenience sampling method and included a hospital setting limited to one northeastern province in China (in and around the city of Dalian), which may limit generalization to other settings. Fifth, the Cronbach a for disclosure score for responsibility was low (0.57); although this suggests that the items within the tool may not be measuring the same underlying construct, Cronbach's alpha has very strict assumptions that are often not met. Finally, our study is observational that can only determine associations and not causality.

\section{CONCLUSION}

Our study shows that depression, anxiety, stigma, and disclosure of HBV infection were associated with low HRQoL in all four domains of the WHOQOL-BREF (physical, psychological, social, and environmental domains), when all psychological factors were included in the model separately. Depression was found to be independently associated with low HRQoL in people living with HBV, when all psychological factors were included in the model simultaneously. Ongoing counseling for people living with $\mathrm{CHB}$ is vital to improving their HRQoL, coping with stress, and improving self-confidence. Psychological health programs must be in place at tertiary medical centers, community hospitals, and ambulatory practices. Our data indicate an urgent need for HCPs and policy-makers to develop and integrate interventions to prevent, screen, and treat psychological morbidity as part of comprehensive care to improve QOL among people living with $\mathrm{CHB}$. 
Received January 3, 2019. Accepted for publication January 31, 2020.

Published online March 2, 2020.

Note: Supplemental tables appear at www.ajtmh.org.

Authors' addresses: Ge Li, Fang-Chi Hsu, Jianzhao Xu, and Avinash Shetty, Wake Forest School of Medicine, Winston-Salem, NC, E-mails: gli@wakehealth.edu, fhsu@wakehealth.edu, jixu@wakehealth.edu, and ashetty@wakehealth.edu. Gongchen Wang, Xia Pei and Bo Zhao, Dalian Sixth People Hospital, Liaoning, China, E-mails: dllygongchenwang@ 126.com, 780950067@qq.com, and bomail@yeah.net.

\section{REFERENCES}

1. World Health Organization, 2018. Hepatitis B Fact sheet. Available at: http://www.who.int/en/news-room/fact-sheets/detail/hepatitis-b. Accessed July 18, 2019.

2. Ren $\mathrm{H}, 2017$. The experience of management of chronic hepatitis B in China. J Viral Hepat 24 (Suppl 1): 4-5.

3. Zhang $S$ et al., 2016. Annual economic burden of hepatitis B virus-related diseases among hospitalized patients in twelve cities in China. J Viral Hepat 23: 202-210.

4. Lam ET, Lam CL, Lai C, Yuen M, Fong DY, So TM, 2009. Healthrelated quality of life of southern Chinese with chronic hepatitis B infection. Health Qual Life Outcomes 7: 52.

5. Spiegel BM et al., 2007. Development and validation of a diseasetargeted quality of life instrument in chronic hepatitis B: the hepatitis B quality of life instrument, version 1.0. Hepatology 46: 113-121.

6. Zhuang G, Zhang M, Liu Y, Guo Y, Wu Q, Zhou K, Ji Z, Zhang X, 2014. Significant impairment of health-related quality of life in mainland Chinese patients with chronic hepatitis B: a crosssectional survey with pair-matched healthy controls. Health Qual Life Outcomes 14: 101.

7. Chao J, Song L, Zhang H, Zhu L, Tian L, Jin H, Liu P, 2013. Effects of comprehensive intervention on health-related quality of life in patients with chronic hepatitis B in China. BMC Health Serv Res 13: 386.

8. Ong SC, Mak B, Aung MO, Li SC, Lim SG, 2009. Health-related quality of life in chronic hepatitis B patients. Hepatology 47: 1108-1117.

9. Liu Y, Zhang S, Zhao Y, Du J, Jin G, Shao S, Lu X, 2016. Development and application of the Chinese (Mainland) version of chronic liver disease questionnaire to assess the health-related quality of life (HRQoL) in patients with chronic hepatitis B. PLOS One 11: e0162763.

10. Xue X, Cai S, Ou H, Zheng C, WuX, 2017. Health-related quality of life in patients with chronic hepatitis $B$ during antiviral treatment and off-treatment. Patient Prefer. Adherence 11: 85-93.

11. Yu L, Wang J, Zhu D, Leng A, Wangen K, 2016. Hepatitis B-related knowledge and vaccination in association with discrimination against hepatitis B in rural China. Hum Vaccin Immunother 12: 70-76.

12. Shi J, Chyun DA, Sun Z, Zhou L, 2013. Assessing the stigma toward chronic carriers of hepatitis B virus: development and validation of a Chinese college students' stigma scale. J Appl Social Psychol 43: E46-E55.

13. Franklin S, Mouliom A, Sinkala E, Kanunga A, Helova A, DionneOdom J, Turan JM, Vinikoor M, 2018. Hepatitis B virus contact disclosure and testing in Lusaka, Zambia: a mixed-methods study. BMJ Open 8: e022522.

14. Keskin G, Gümüs AB, Orgun F, 2013. Quality of life, depression, and anxiety among hepatitis B patients. Gastroenterol Nurs 36 : 346-356.

15. Duan Z, Kong Y, Zhang J, Guo H, 2012. Psychological comorbidities in Chinese patients with acute-on-chronic liver failure. Gen Hosp Psychiatr 34: 276-281.

16. Jinlin $\mathrm{H}$ et al., 2017. Guideline of prevention and treatment for chronic hepatitis B. J Clin Transl Hepatol 5: 297-318.

17. Chinese Society of Hepatology and Chinese Society of Infectious Diseases, Chinese Medical Association, 2011. Guideline of prevention and treatment for chronic hepatitis B. Zhonghua Gan Zang Bing Za Zhi 19: 13-24.

18. Gao K, Su M, Sweet J, Calabrese JR, 2019. Correlation between depression/anxiety symptom severity and quality of life in patients with major depressive disorder or bipolar disorder. $J$ Affect Disord 244: 9-15.

19. Li D, Tang T, Patterson M, Ho M, Heathcote J, Shah H, 2012. The impact of hepatitis $B$ knowledge and stigma on screening in Canadian Chinese persons. Can J Gastroenterol 26: 597-602.

20. Derlega VJ, Lovejoy D, Winstead BA, 1998. Personal accounts of disclosing and concealing HIV-positive test results: weighing the benefits and risks. Derlega V, Barbee A, eds. HIV Infection and Social Interaction. Newbury Park, CA: Sage, 147-164.

21. Serovich JM, Mosack KE, 2003. Reasons for HIV disclosure or nondisclosure to casual sexual partners. AIDS Educ Prev 15: 70-80.

22. Beck AT, Ward CH, Mendelson M, Mock J, Erbaugh J, 1961. An inventory for measuring depression. Arch Gen Psychiatr 4: 561-571.

23. Beck AT, Epstein N, Brown G, Steer RA, 1988. An inventory for measuring clinical anxiety: psychometric properties. $J$ Consult Clin Psychol 56: 893-897.

24. Liang Y, Wang L, Zhang J, 2018. Factor structure and psychometric properties of Chinese version of Beck anxiety inventory in Chinese doctors. J Health Psychol 23: 657-666.

25. Skevington SM, McCrate FM, 2012. Expecting a good quality of life in health: assessing people with diverse diseases and conditions using the WHOQOL-BREF. Health Expect 15: 49-62.

26. Liang WM, Chang CH, Yeh YC, Shy HY, Chen HW, Lin MR, 2009. Psychomeric evaluation of the WHOQOL-BREF in communitydwelling older people in Taiwan using Rasch analysis. Qual Life Res 18: 605-618.

27. World Health Organization, 2004. WHO Quality of Life-BREF (WHOQOL-BREF). Available at: http://www.who.int/substance_ abuse/research_tools/whoqolbref/en/.

28. Fábregas BC, de Ávila RE, Faria MN, Moura AS, Carmo RA, Teixeira AL, 2013. Health related quality of life among patients with chronic hepatitis C: a cross-sectional study of sociodemographic, psychopathological and psychiatric determinants. Braz J Infect Dis 17: 633-639.

29. Ozkan M et al., 2006. Psychiatric morbidity and its effect on the quality of life of patients with chronic hepatitis $B$ and hepatitis $C$. Int J Psychiatr Med 36: 283-297.

30. Hu CG, Yuan GS, Huang HP, Liu JW, Zhou YC, Ren YY, Li Y, Tan WJ, Su ML, Zhou YP, 2017. Depression and anxiety caused by pegylated interferon treatment in patients with chronic hepatitis $B$ and the therapeutic effects of escitalopram and alprazolam. Nan Fang Yi Ke Da Xue Xue Bao 37: 1201-1205.

31. Han B et al., 2018. The experience of discrimination of individuals living with chronic hepatitis $B$ in four provinces of China. PLoS One 13: e0195455.

32. Huang J, Guan ML, Balch J, Wu E, Rao H, Lin A, Wei, Lok AS, 2016. Survey of hepatitis $B$ knowledge and stigma among chronically infected patients and uninfected persons in Beijing, China. Liver Int 36: 1595-1603.

33. Cotler SJ, Cotler S, Xie H, Luc BJ, Layden TJ, Wong SS, 2012. Characterizing hepatitis B stigma in Chinese immigrants. J Viral Hepat 19: 147-152.

34. Wu CA, Lin SY, So SK, Chang ET, 2007. Hepatitis B and liver cancer knowledge and preventive practices among Asian Americans in the San Francisco, Bay Area, California. Asian Pac $J$ Cancer Prev 8: 127-134.

35. Taylor VM, Coronodo G, Acorda E, Teh C, Tu SP, Yasui Y, Bastani R, Hislop TG, 2008. Development of an ESL curriculum to educate Chinese immigrants about hepatitis B. J Community Health 33: 217-224.

36. Li Y, Chen WW, Wei L, Xie YX, Wang LF, Fu JL, Wang LF, 2017. A survey of knowledge about hepatitis $B$ among new military recruits in China. Mil Med Res 4: 2.

37. Chao J, Chang E, So S, 2010. Hepatitis B and liver cancer knowledge and practices among healthcare and public health professional in China: a cross-sectional study. BMC Public Health 10: 98.

38. Yang T, Wu M-C, 2011. Discrimination against hepatitis B carriers in China. Lancet 378: 1059.

39. Leng A, Li Y, Wangen KR, Nicholas S, Maitland E, Wang J, 2016. Hepatitis $B$ discrimination in everyday life by rural migrant workers in Beijing. Hum Vaccine Immunother 12: 1164-1171. 